\title{
Periodic layers of a dodecagonal quasicrystal and a floating hexagonal crystal in sedimentation-diffusion equilibria of colloids
}

Harini Pattabhiraman, and Marjolein Dijkstra

Citation: The Journal of Chemical Physics 147, 104902 (2017);

View online: https://doi.org/10.1063/1.4993521

View Table of Contents: http://aip.scitation.org/toc/jcp/147/10

Published by the American Institute of Physics

\section{Articles you may be interested in}

Phase behaviour of quasicrystal forming systems of core-corona particles The Journal of Chemical Physics 146, 114901 (2017); 10.1063/1.4977934

Phase and vacancy behaviour of hard "slanted" cubes

The Journal of Chemical Physics 147, 124501 (2017); 10.1063/1.5001483

The effect of finite pore length on ion structure and charging

The Journal of Chemical Physics 147, 104708 (2017); 10.1063/1.4986346

Diffusion and interactions of point defects in hard-sphere crystals

The Journal of Chemical Physics 146, 244905 (2017); 10.1063/1.4990416

Liquid bridging of cylindrical colloids in near-critical solvents

The Journal of Chemical Physics 147, 104701 (2017); 10.1063/1.4986149

Variation of ionic conductivity in a plastic-crystalline mixture

The Journal of Chemical Physics 147, 104502 (2017); 10.1063/1.5001946

\section{AIP | The Joumal of Chemical Physics}




\title{
Periodic layers of a dodecagonal quasicrystal and a floating hexagonal crystal in sedimentation-diffusion equilibria of colloids
}

\author{
Harini Pattabhiraman a) and Marjolein Dijkstrab) \\ Department of Physics, Soft Condensed Matter, Debye Institute for Nanomaterials Science, Utrecht University, \\ Princetonplein 5, 3584 CC Utrecht, The Netherlands
}

(Received 29 June 2017; accepted 30 August 2017; published online 14 September 2017)

\begin{abstract}
We investigate the behaviour of a system of colloidal particles interacting with a hard-core and a repulsive square shoulder potential under the influence of a gravitational field using event-driven Brownian dynamics simulations. We use a fixed square shoulder diameter equal to 1.4 times the hardcore diameter of the colloids, for which we have previously calculated the equilibrium phase diagram considering two-dimensional disks [H. Pattabhiraman et al., J. Chem. Phys. 143, 164905 (2015) and H. Pattabhiraman and M. Dijkstra, J. Phys.: Condens. Matter 20, 094003 (2017)]. The parameters in the simulations are chosen such that the pressure at the bottom of the sediment facilitates the formation of phases in accordance with the calculated phase diagram of the two-dimensional system. It is surprising that we observe the formation of layers with dodecagonal, square, and hexagonal symmetries at the relevant pressures in the three-dimensional sedimentation column. In addition, we also observe a re-entrant behaviour exhibited by the colloidal fluid phase, engulfing a hexagonal crystal phase, in the sedimentation column. In other words, a floating crystal is formed between the colloidal fluid regions. Published by AIP Publishing. [http://dx.doi.org/10.1063/1.4993521]
\end{abstract}

\section{INTRODUCTION}

In the case of colloidal suspensions consisting of particles with sizes on the order of micro-meters, the effect of the gravitational force is not negligible. Under these conditions, the gravitational energy and the thermal energy of the colloids are comparable. This leads to the formation of a spatially inhomogeneous suspension in which the density of the particles varies along the height of the suspension. The inhomogeneous distribution of the colloidal particles along the height under the influence of gravity is termed as sedimentation.

As a result of this inhomogeneous density distribution in colloidal suspensions, the particles at the bottom of the sediment can crystallise when they reach a certain density. Experimentally, sedimentation is regarded as a prevalent tool to extract information regarding the equilibrium phase behaviour of the system. For example, the measured concentration profiles can be inverted to obtain the osmotic equation of state. Sedimentation processes can also be used the other way around, i.e., they can be used to validate the theoretically calculated equilibrium phase behaviour of a system and thereby its bulk phase behaviour. For example, a system of hard spheres has been a model system for sedimentation studies. This system exhibits a phase behaviour characterised by a fluid phase at low densities and a face-centered cubic phase at high densities. This behaviour, which was earlier theoretically predicted, ${ }^{1}$ has later been corroborated by sedimentation studies. ${ }^{2}$

Sedimentation behaviour of various charged particles, ${ }^{3}$ mixtures of hard particles, ${ }^{4}$ and particles of different shapes ${ }^{5}$ which result in the formation of various colloidal crystals has

\footnotetext{
a)h.pattabhiraman@uu.nl

b)m.dijkstra@uu.nl
}

been extensively studied. However, similar studies involving the formation of quasicrystals (QCs) by sedimentation has received less attention. Quasicrystals are solids with longrange orientational order and no periodicity and may exhibit intriguing properties including the formation of photonic band gaps. ${ }^{6-11}$ Hence, the experimental realisation of quasicrystals is a topic of intense research. ${ }^{12-18}$

In our previous studies, ${ }^{19,20}$ we have theoretically calculated the phase diagram of a two-dimensional system of particles interacting with a hard-core and repulsive square shoulder (HCSS) potential. At a shoulder width of 1.4 times the hard core diameter of the particle, we find a stable randomtiling dodecagonal quasicrystal. In this work, we investigate how the phase behaviour in a two-dimensional HCSS system extends to three-dimensional systems and whether or not multiple layers of this quasicrystal can be self-assembled by sedimentation in three dimensions.

This paper is organised as follows. In Sec. II, we present the simulation and analysis methods used. In Sec. III, we individually discuss the formation of layers with various symmetries, and finally we present the conclusions and the direction of future studies in Sec. IV.

\section{METHODS}

We first explain the simulation model and computational methods used for this study in Sec. II A and then give an account of the analysis methods in Sec. II B.

\section{A. Computational methodology}

We perform Event-Driven Brownian Dynamics (EDBD) simulations of $N$ spherical particles of diameter $\sigma_{H S}$ and buoyant mass $m$ interacting with the HCSS potential in the NVT 
ensemble. The HCSS potential can be written as a sum of a hard-sphere potential $V_{\mathrm{HS}}(r)$ and a square-shoulder potential $V_{\mathrm{SS}}(r)$, i.e.,

$$
V_{\mathrm{HCSS}}(r)=V_{\mathrm{HS}}(r)+V_{\mathrm{SS}}(r),
$$

where

$$
V_{\mathrm{HS}}(r)= \begin{cases}\infty, & r \leq \sigma_{H S} \\ 0, & r>\sigma_{H S}\end{cases}
$$

and

$$
V_{\mathrm{SS}}(r)=\left\{\begin{array}{ll}
\epsilon, & r \leq \delta \\
0, & r>\delta
\end{array},\right.
$$

where $r$ is the interparticle center-of-mass distance and $\epsilon>0$ is the height of the square shoulder.

In the EDBD method, a sequence of collision events involving only two particles at any given instant is computed. During the simulation, the velocities of the particles are randomly adjusted at regular intervals $\Delta t$ as

$$
\mathbf{v}(t+\Delta t)=\alpha_{t} \mathbf{v}(t)+\beta_{t} \mathbf{v}_{R}(t),
$$

where $\mathbf{v}(t)$ and $\mathbf{v}(t+\Delta t)$ are, respectively, the velocities of the particles before and after the stochastic velocity adjustment, $\mathbf{v}_{R}(t)$ is a 3-D Gaussian variable with a mean of 0 and variance of $k_{B} T / m$, with $k_{B}$ as the Boltzmann constant and $T$ as the temperature. Further, $\alpha_{t}$ has a value $1 / \sqrt{2}$ with a probability $v \Delta t$ and 1 otherwise. The temperature is kept constant by setting $\beta_{t}=\sqrt{1-\alpha_{t}^{2}}$. In accordance to previous EDBD simulations, ${ }^{21,22}$ we set $v$ to $10 \tau_{M D}^{-1}$ and $\Delta t$ to $0.01 \tau_{M D}$, where $\tau_{M D}$ is the unit of time of an event-driven molecular dynamics simulation given as $\tau_{M D}=\sqrt{m / k_{B} T} \sigma_{H S}$.

The present simulation method is an event driven molecular dynamics (EDMD) method which is extended with Brownian motion. Previous experiments and simulations show that the structure of the crystalline sediment depends strongly on the Brownian time, the sedimentation time, and the initial volume fraction..$^{21,23}$ The faster the sedimentation, the higher the initial volume fraction, and the less time the system has available for equilibration during sedimentation and the crystalline sediment will become more defective. Brownian motion is, thus, important for equilibration during sedimentation. In this case, the Brownian time is set by $\alpha_{t}$ and $v$. It is convenient to define a dimensionless Peclet number $g^{*}$ that is equal to the inverse gravitational length and a dimensionless particle flux as defined by the ratio of the Brownian time and sedimentation time. In our simulations, the Peclet number ranges from 1 to 5 , whereas the particle flux is in the range of 0.01-0.05, where previous simulations and experiments show that there is ample time for equilibration and least defective crystalline sediments are obtained. ${ }^{21,23}$

The simulation box of volume $V$ has a square cross section of area $A$ and is elongated in the $z$-direction. Periodic boundary conditions are applied along the cross section. In the elongated direction, the particles are confined between two smooth hard walls at $z=0$ and $z=H$ with $H$ as the height of the sedimentation column. The height $H$ is chosen such that the density at $z=\left(H-\sigma_{H S} / 2\right)$ is sufficiently small, which allows us to consider the system to be infinitely long in the $z$-direction. We perform simulations starting with a non-overlapping isotropic fluid state filling the entire sedimentation column with packing fraction $\eta=0.01$. To mimic sedimentation experiments, these particles are further subjected to a gravitational field, which is expressed as an external potential $\phi(z)$ written as

$$
\phi(z)= \begin{cases}m g z, & \sigma_{H S} / 2 \leq z \leq\left(H-\sigma_{H S} / 2\right) \\ \infty, & \text { otherwise }\end{cases}
$$

where $g$ is the acceleration due to gravity and $z$ is the vertical coordinate of the particle. The effect of the gravitational field on the particles is quantified in terms of the gravitational Peclet number defined as $g^{*}=m g \sigma_{H S} / k_{B} T$.

In this work, we scrutinise the kinetic formation of the thermodynamic stable phases described for the twodimensional HCSS system with $\delta=1.40 \sigma_{H D}$, where $\sigma_{H D}$ is the hard-disk diameter, as used in our previous works. ${ }^{19,24}$ To do so, we perform simulations such that the pressure measured at the bottom of the sedimentation column, i.e., at $z=0$, corresponds to the region of stability of a particular phase in the 2-D phase diagram. This pressure is calculated as $P^{*}=\beta P(z$ $=0) \sigma_{H S}^{3}=g^{*} \cdot \rho_{A}^{*}$, where $\rho_{A}^{*}$ is the mean areal density defined as the number of particles at the bottom of the sample per area $\rho_{A}^{*}=N \sigma_{H S}^{2} / A$, and we compare $P^{*}$ with $P_{2 D}^{*}=\beta P \sigma_{H D}^{2}$ directly. However, we note that the direct comparison of pressures between two- and three-dimensional systems may not always hold true. For example, the fluid (hexatic)-solid transition in the case of hard disks is at $P_{2 D}^{*}=9.17,{ }^{25}$ whereas that for hard spheres is at $\beta P \sigma_{H S}^{3}=11.57 .{ }^{26}$ It is $\operatorname{good}$ to remind the readers that we use a three-dimensional system of spheres here to validate the phase behaviour of a two-dimensional system of disks. The underlying reason for this will be explained in Secs. II B and III A-III C.

The phases considered in this study are dodecagonal quasicrystal, square, low-density hexagonal, and fluid phases. We especially focus on the possibility of the formation of the quasicrystal and, thus, consider the cross section to be squares with sides of length $58 \sigma_{H S}$, which can accommodate a randomtiling dodecagonal quasicrystal at a density $\rho^{*}=1.07$, similar to that used in Ref. 19. The list of parameters used to simulate the different phases is given in Table I. The corresponding values of pressures at the bottom $P_{2 D}^{*}=\beta P(z=0) \sigma_{H S}^{3}$ are marked in the phase diagram given in Fig. 1. As a supplementary study, we use two parameter sets having different Peclet numbers to simulate the quasicrystal. As mentioned above, a higher value of the Peclet number results in a condition of high settling rate of the particles, i.e., the particles do not have enough time to rearrange and equilibrate, and vice versa. Using systems with different Peclet numbers allows us

TABLE I. System parameters used in the EDBD simulations of a HCSS system with $\delta=1.40 \sigma_{H S}$ under gravity.

\begin{tabular}{lcccl}
\hline \hline$N$ & $k_{B} T / \epsilon$ & $g^{*}$ & $\beta P(z=0) \sigma_{H S}^{3}$ & Stable phase at bottom \\
\hline $5 \times 10^{4}$ & 0.25 & 2.00 & 30.0 & Quasicrystal \\
$2 \times 10^{4}$ & 0.25 & 5.00 & 30.0 & Quasicrystal \\
$2 \times 10^{4}$ & 0.25 & 4.00 & 23.8 & Square \\
$5 \times 10^{4}$ & 0.15 & 0.67 & 10.0 & Low-density hexagonal \\
$1 \times 10^{5}$ & 0.15 & 0.67 & 20.0 & Fluid \\
\hline \hline
\end{tabular}




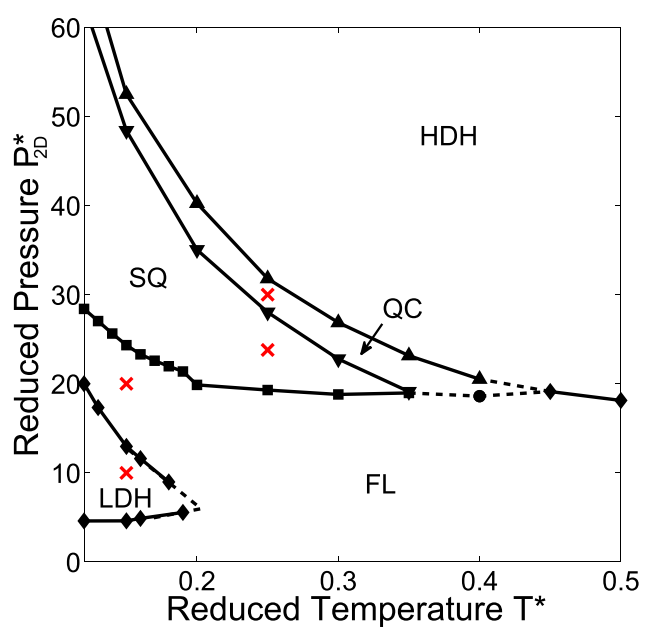

FIG. 1. Phase diagram in the (reduced) pressure-temperature plane for a twodimensional HCSS system with shoulder width $\delta=1.40 \sigma_{H D}$. The reduced quantities are defined as $P_{2 D}^{*}=\beta P \sigma_{H D}^{2}$ and $T^{*}=k_{B} T / \epsilon$. The phases marked are fluid (FL), low-density hexagonal (LDH), square (SQ), dodecagonal quasicrystal (QC), and high-density hexagonal (HDH). The crosses denote the state points corresponding to the pressures at the bottom of the sediment.

to study the effect of the settling rate on the formation of the quasicrystal.

\section{B. Structural analysis}

In order to characterise the different phases, we employ an analysis method that is two-dimensional in nature since the phases observed in the sedimentation column have a layered structure and resemble the phases observed for the HCSS system in 2-D. Specifically, we first identify different layers of the sediment and then carry out the following analysis procedure in these layers. We construct the polygonal tiling of the layer and calculate the two-dimensional $m$-fold bond orientational order parameter (BOO) of each particle $j$ in layer $l, \chi_{m}^{l}(j)$, and the average BOO of each layer $\chi_{m}^{l}$ as explained in Ref. 20.

The polygonal tiling of each layer is constructed by drawing bonds between the neighbouring particles of each particle $j$, which are at a center-of-mass distance smaller than the square shoulder diameter $\delta$ from particle $j$.

We, then, calculate the $m$-fold BOO of each particle $j$ in layer $l$ as

$$
\chi_{m}^{l}(j)=\left|\frac{1}{N_{B}(j)} \sum_{k=1}^{N_{B}(j)} \exp \left(i m \theta_{\mathbf{r}_{j k}}\right)\right|^{2},
$$

where $m$ is the symmetry of interest, $\mathbf{r}_{j k}$ is the center-of-mass distance vector between two neighbours $j$ and $k, \theta_{\mathbf{r}_{j k}}$ is the angle

TABLE II. Method of classification of particle $j$ belonging to layer $l$ according to its bond orientational order (BOO) $\chi_{m}^{l}(j)$.

\begin{tabular}{lcc}
\hline \hline Symmetry & BOO conditions & Colour \\
\hline Fluid/other (OT) & $\chi_{4}^{l}(j), \chi_{6}^{l}(j), \chi_{12}^{l}(j)<0.5$ & Orange \\
Crystal & $\chi_{4}^{l}(j), \chi_{6}^{l}(j), \chi_{12}^{l}(j)>0.5$ & \\
Square (SQ) & $\chi_{4}^{l}(j)>\chi_{6}^{l}(j), \chi_{12}^{l}(j)$ & Purple \\
Hexagonal (HX) & $\chi_{6}^{l}(j)>\chi_{4}^{l}(j), \chi_{12}^{l}(j)$ & Green \\
Dodecagonal (QC) & $\chi_{12}^{l}(j)>\chi_{4}^{l}(j), \chi_{6}^{l}(j)$ & Red \\
\hline \hline
\end{tabular}

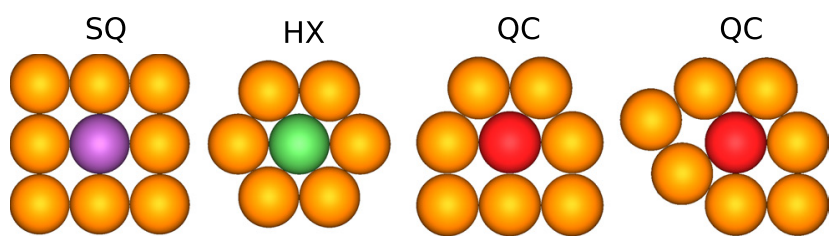

FIG. 2. Colour scheme for classes of particles based on the BOO classification described in Table II.

between $\mathbf{r}_{j k}$ and an arbitrary axis, and $N_{B}(j)$ is the number of neighbours of particle $j$ in the same layer. For each particle $j$, we calculate $\chi_{4}^{l}(j), \chi_{6}^{l}(j)$, and $\chi_{12}^{l}(j)$, respectively, representing square, hexagonal, and dodecagonal symmetries.

The particles are classified based on their BOO according to the method given in Table II. We consider a particle to be fluid-like if each of the three $\chi_{m}^{l}(j)$ is less than 0.5 . On the other hand, if each of $\chi_{m}^{l}(j)$ is greater than 0.5 , then a particle is said to have symmetry $m 1$ if $\chi_{m 1}^{l}(j)$ is greater than the other two, namely, $\chi_{m 2}^{l}(j)$ and $\chi_{m 3}^{l}(j)$. Further, we identify and colour the particles according to the following scheme: particles of square symmetry in purple, those of hexagonal in green, dodecagonal in red, and fluid-like in orange as shown in Fig. 2.

After calculating the $\mathrm{BOO}$ of each particle, the average $\mathrm{BOO}$ of each layer is then evaluated as ${ }^{27}$

$$
\chi_{m}^{l}=\frac{1}{N_{l}} \sum_{j=1}^{N_{l}} \chi_{m}^{l}(j),
$$

where $N_{l}$ is the number of particles in each layer.

\section{RESULTS AND DISCUSSION}

In this section, we consider individually the different sedimentation simulations carried out to obtain the various stable phases calculated for the two-dimensional HCSS system.

\section{A. Formation of layers with dodecagonal symmetry}

We start with the formation of layers with dodecagonal symmetry. In this section, we present the sedimentation simulations using two different Peclet numbers in order to assess the effect of the settling rate on the formation of the quasicrystal. We first compare the formation of the quasicrystal formed in these simulations and then analyse the driving force behind the formation of these layers. Finally, we review the validity of the phase diagram given in Fig. 1 by comparing the phases formed in the sedimentation column. The dodecagonal quasicrystal (QC) which, as seen in the phase diagram, is sandwiched between two periodic crystal phases with square and hexagonal symmetries. Thus, it is interesting to note if and how the interfaces between the quasicrystal and the periodic crystals are formed in the sedimentation column.

We first present a typical configuration of the sediment forming quasicrystalline layers in Fig. 3. The panels on the left correspond to simulations with a Peclet number $g^{*}=5.0$ and those on the right are obtained for $g^{*}=2.0$. The particles here are coloured according to the convention explained in Fig. 2. We notice the formation of about two quasicrystalline layers for $g^{*}=5.0$ and about four quasicrystalline layers for $g^{*}=2.0$. This difference in the number of layers is due to a 

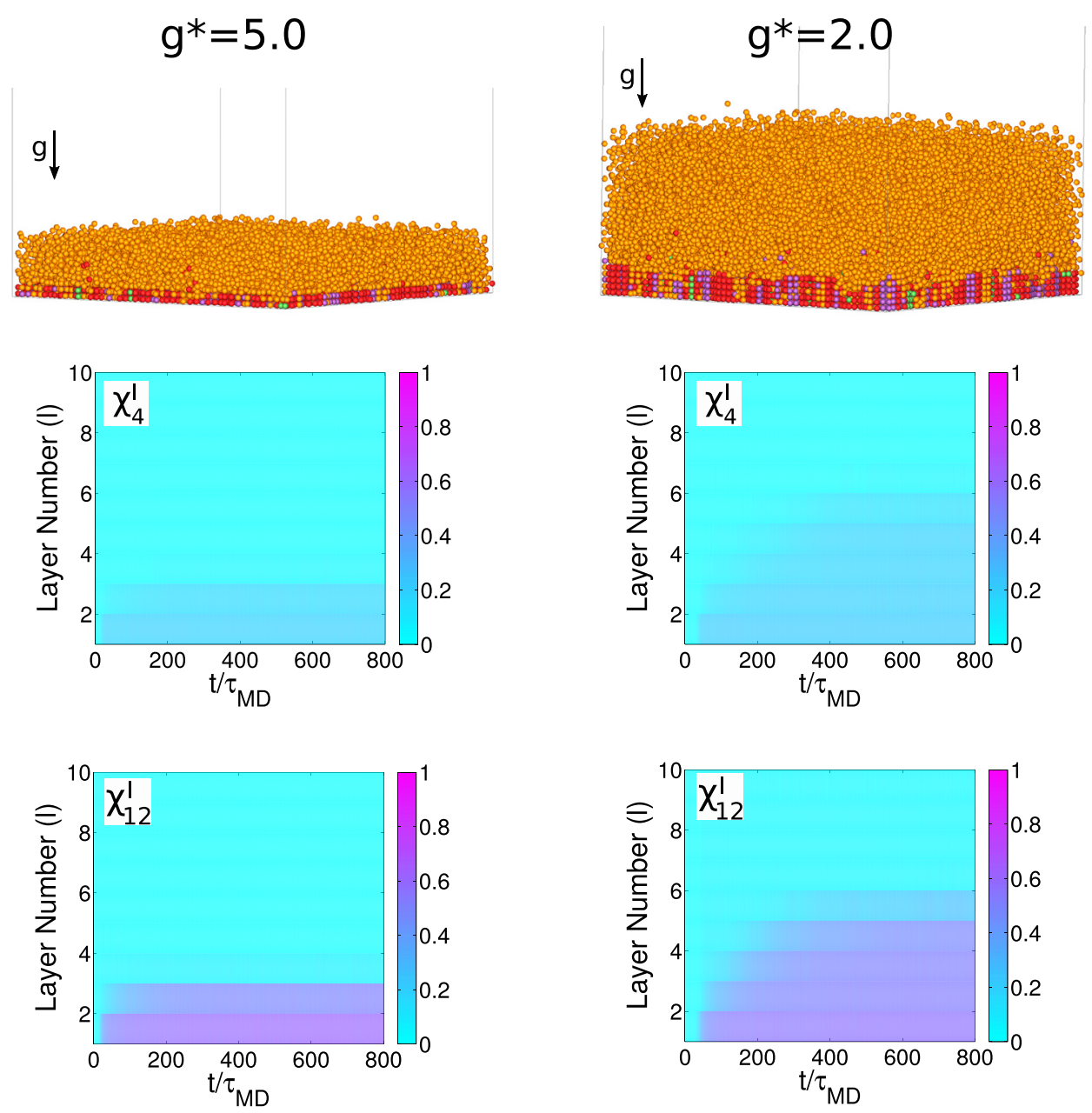

FIG. 3. Comparison of the quasicrystal (QC) sediment formed for Peclet numbers $g^{*}=5.0$ (left) and 2.0 (right). Side view of a configuration of the sedimentation column obtained at $t / \tau_{M D}$ $=800$ (top). The particles are coloured according to their individual BOO: quasicrystal (red), square (purple), hexagonal (green), and fluid (orange). The $m$-fold BOO of each layer with time calculated for symmetries $m=4$ (middle) and 12 (bottom) showing, respectively, the formation of layers with square and dodecagonal symmetries.

difference in the height range that corresponds to the pressure range of the stable quasicrystal phase. This height range decreases with increasing $g^{*}$. Additionally, we observe that most of the particles seen in these layers are coloured either in purple or red which, respectively, represent square or dodecagonal symmetries. Therefore, we follow the dynamics of the formation of these layers by calculating the BOO $\chi_{4}^{l}$ and $\chi_{12}^{l}$ of each layer as a function of time. The time evolution of $\chi_{4}^{l}$ is given in the middle panel in Fig. 3 and that of $\chi_{12}^{l}$ is given at the bottom. In these time evolution heat maps, the time scale $t / \tau_{M D}$ is plotted on the horizontal axis and the layer number is plotted along the vertical axis.

We make the following observations from these plots: (1) In both cases, the value of $\chi_{12}^{l}$ is higher than that of $\chi_{4}^{l}$, which confirms the dodecagonal symmetry of these layers. (2) With increasing time, we find that the fraction of fluid in the sedimentation column decreases, as seen by the receding blue region in these plots. Alternatively, this means that more crystalline layers are formed with time. (3) The value of $\chi_{12}^{l}$ at a given time decreases as we go up in the sediment indicating that the layers on the top are more fluid-like than the bottom layers. (4) Finally, we see that $\chi_{12}^{l}$ obtained for the sediment at higher Peclet numbers is larger than that at lower Peclet numbers. This is counter-intuitive as this suggests that faster settling of the particles result in the formation of a less-defective quasicrystal.
We investigate this further by plotting the polygonal tiling constructed for the bottom two layers as a function of time for both the sediments. The top view of these tilings is given in Fig. 4 for $g^{*}=5.0$ and in Fig. 5 for $g^{*}=2.0$. Two striking features are conspicuous from these polygonal tilings. First, there are large portions of connected square tilings in the sediment obtained for the lower Peclet number, while the square tilings are more uniformly distributed in the case of the high Peclet number sediment. Second, the position of the tiles in the first and second layers seems to be on top of each other. Let us now evaluate each of these observations separately.

Firstly, we analyze the tilings and quantify the square tiles by calculating the ratio of areas occupied by the square tiles to those of the triangle tiles. This also relates to the squaretriangle tiling description of a dodecagonal quasicrystal, where the maximum entropy of the tiling corresponds to equal areas of squares and triangles. ${ }^{28-30}$ In the current sediments, we find that the ratio of the areas of squares to triangles for $g^{*}=2.0$ is $1.40 \pm 0.05$ and for $g^{*}=5.0$ is $1.15 \pm 0.03$. In other words, in both cases, there are more squares formed than in an ideal dodecagonal tiling. This excess of squares is larger for the low Peclet number sediment. This can be explained as follows. A lower Peclet number refers to a lower rate of sedimentation and, thus, a longer relaxation time for the particles to rearrange. The density at the bottom of the sample increases slowly since the beginning of the sedimentation simulation, as more and 
layer 1
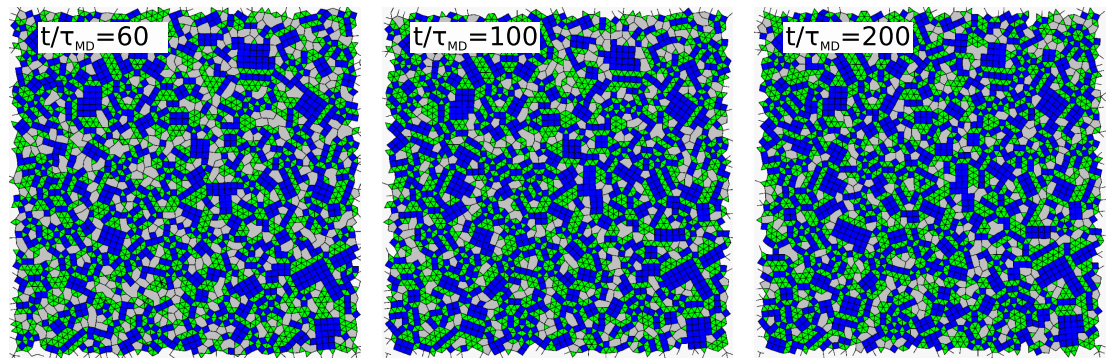

layer 2

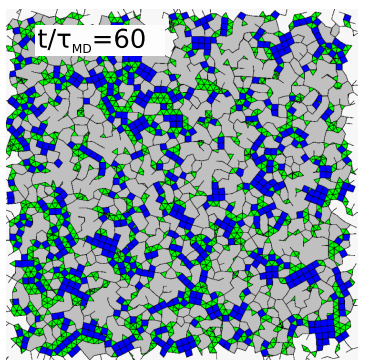

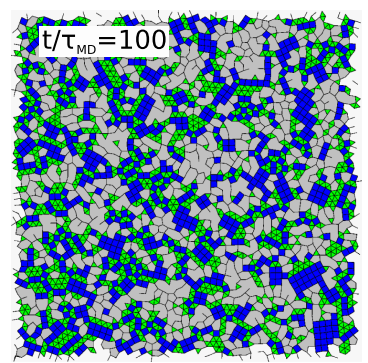

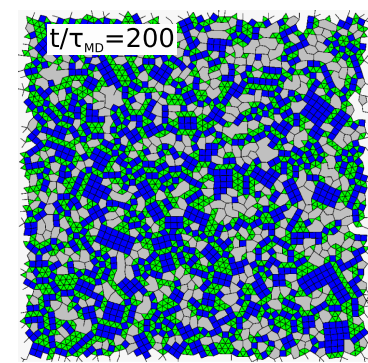

FIG. 4. Polygonal tilings as obtained from particle configurations of the first (top) and second (bottom) layers of the QC sediment for the Peclet number $g^{*}$ $=5.0$ showing the formation of the quasicrystal for varying times $t / \tau_{M D}$ as labeled. The triangle, square, and defect tiles are, respectively, coloured in blue, green, and gray. more particles descend through the column. This means that the density at the bottom layer first reaches the value where a square phase is found to be stable. Thus, nuclei consisting of particles with square symmetry start to form. This can be observed in the snapshot of the second layer at $t / \tau_{M D}=60$ at both Peclet numbers. However, in the case of the sediment with lower Peclet numbers, these nuclei have enough time to aggregate and, thus, start forming larger square structures. On the other hand, the square nuclei of the fast settling sediment does not have enough time to rearrange and, thus, are spread all over the area. Also, a larger settling rate means that the density corresponding to the formation of the quasicrystal is reached faster at the bottom of the sediment. This again means that lesser amount of square tiles are formed in the sediment with higher Peclet numbers. Because of these reasons, the sediment with a high Peclet number results in the formation of a lesser number of square tiles and also lower aggregation of these tiles than the slow settling sediment.

Now, let us examine the observation regarding the position of the tiles of the second layer directly on top of the first layer. This, in essence, refers to the formation of quasicrystal layers which are periodic in the third direction. To assess this, we show the top view of the particles of the first and second layers obtained for the fast settling sediment in Fig. 6. Here, the particles in the first layer are represented as filled blue circles, while those of the second layer are represented as open black circles. In the figure, we observe that a majority of the blue particles are enclosed in a black circle. In other words, the position of the particles of the second layer is on top of the first layer, which confirms that these structures are periodic in the third dimension. This leads us to the question of why do the particles of the second layer not position themselves in the voids of the particles of the bottom layer, as is expected from simulations involving gravitational fields.

The answer to this question lies in the fact that the interaction potential between the particles is purely repulsive and has two-length scales. We explain this further by using a schematic description in Fig. 7. The colour coding of the particles is the same as described above; the particles in the first layer are represented as filled blue circles, while those of the second layer layer 1
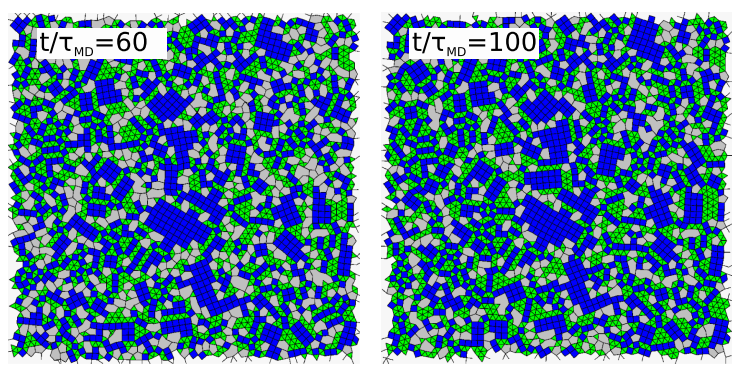

layer 2

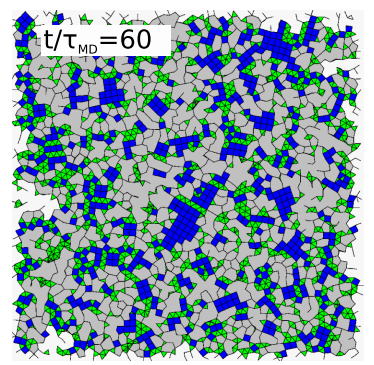

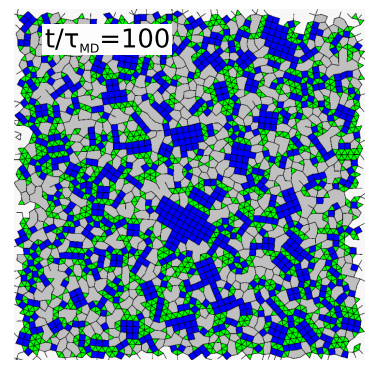
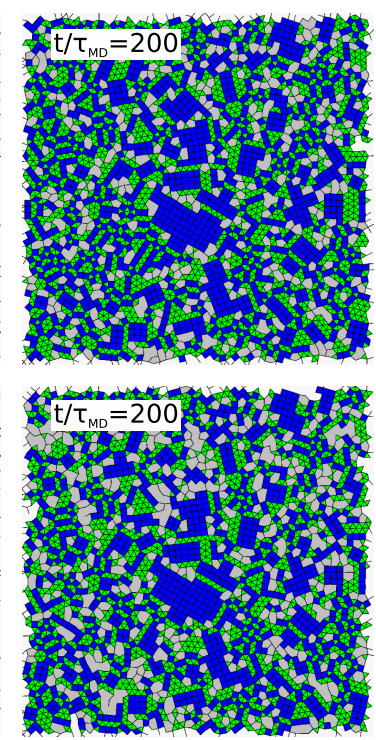

FIG. 5. Polygonal tilings as obtained from particle configurations of the first (top) and second (bottom) layers of the QC sediment for the Peclet number $g^{*}$ $=2.0$ showing the formation of the quasicrystal for varying times $t / \tau_{M D}$ as labeled. The triangle, square, and defect tiles are, respectively, coloured in blue, green, and gray. 


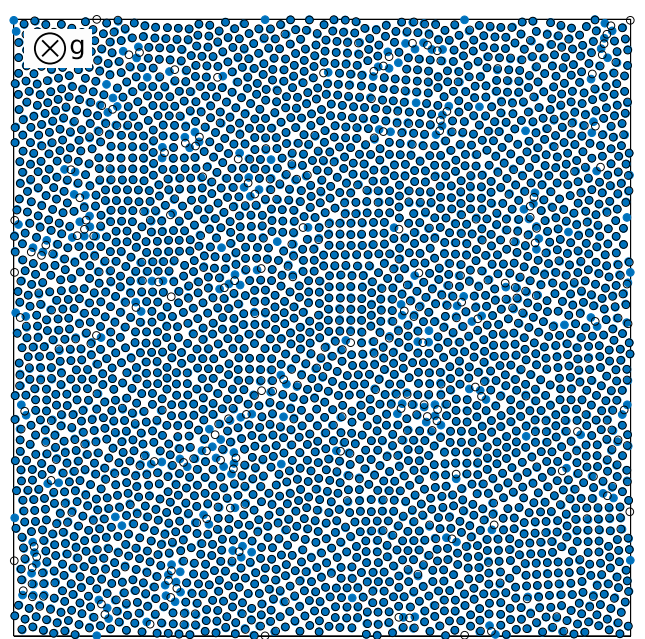

layer $1 \bigcirc \quad$ layer $2 \bigcirc$

FIG. 6. Top view of the center-of-masses of particle configurations of the first and second layers obtained for the QC sediment with the Peclet number $g^{*}$ $=5.0$ at $t / \tau_{M D}=800$. The particles in the bottom layer are plotted as filled circles in blue and the particles in the top layer are represented as open black circles. The gravitational field points into the plane of the paper as marked in the top-left corner.

are represented as open black circles. On the top, we represent the situation where the particles of the second layer fall in the voids of the particles of the first layer. The front view of the sediment is shown on the left and the top view is shown on the right. The present case of the periodic layers is shown at the bottom. First, let us look at the scenario with alternating layers, i.e., the particles of the second layer are in the voids of those in the first layer. In this case, we see that each particle in the second layer, in addition to its nearest neighbour in the second layer, has three neighbours in the first layer. In contrast, in the case of the present situation of periodic layers (shown at the bottom in Fig. 7), each particle of the second layer has only a single neighbouring particle in the bottom layer. The same also applies for the third layer on top of the second, thereby doubling the number of neighbours of each particle. This reduction in the number of neighbours is translated as a reduction in the energy of the particle and, thus, of the entire system. This configuration is favoured, if this reduction in energy is more than the increase in potential energy of

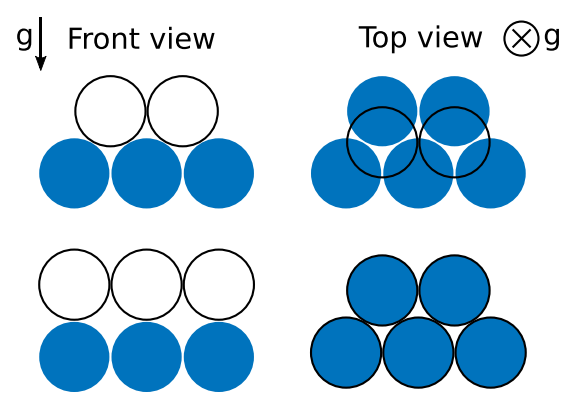

FIG. 7. Schematic representation of the energetic driving force behind the formation of layers with particles on top of each other. The particles in the bottom layer are plotted as filled circles in blue and the particles in the top layer are represented as open black circles. The direction of the gravitational field in each case in also marked on the top.

the particle for being at a higher position in the sedimentation column, i.e., at a higher position on top of a particle instead of a lower position in the void. This happens to be the case in our simulations, which leads to the formation of these periodic layers. This also means that these layers are stabilised by gravity. Finally, this formation of a layered structure justifies the validation of the phase behaviour of a two-dimensional system of disks using a three-dimensional system of spheres.

Finally, we proceed to the validation of the phase diagram in terms of the phases formed along the height of the sedimentation column, which corresponds to a decrease in pressure. To analyze this, we plot the pressure and density profiles along the height of the sedimentation column calculated for both Peclet numbers in Fig. 8. The pressure and density profiles corresponding to the high Peclet number are on the left and those of the low Peclet number are on the right. In these plots, we explicitly mark the pressure boundaries denoting the stability of each phase as obtained from the 2-D phase diagram. From the density profiles, we observe that the formation of two (quasi)-crystalline layers at a high Peclet number and five at a low Peclet number. Beyond this pressure, we find the presence of an isotropic fluid, which agrees well with the phase diagram prediction. However, the puzzling part is the absence of the square phase.

From the density plots, we see that the crystalline layers are formed in the sediment for pressures corresponding to the stable QC or SQ regime in the phase diagram. However, all

$$
g^{*}=5.0
$$
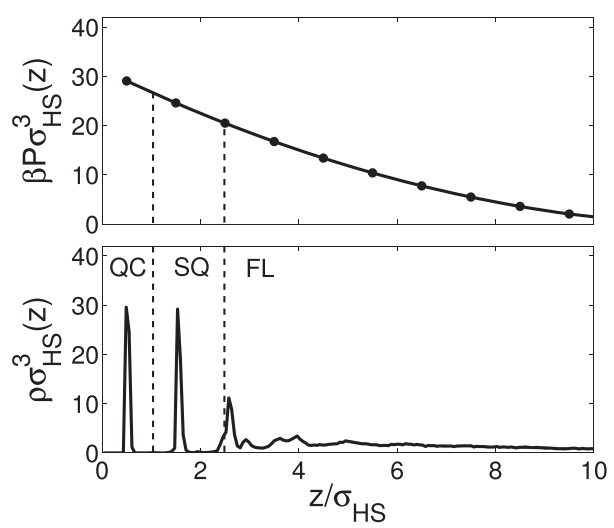

$g^{*}=2.0$

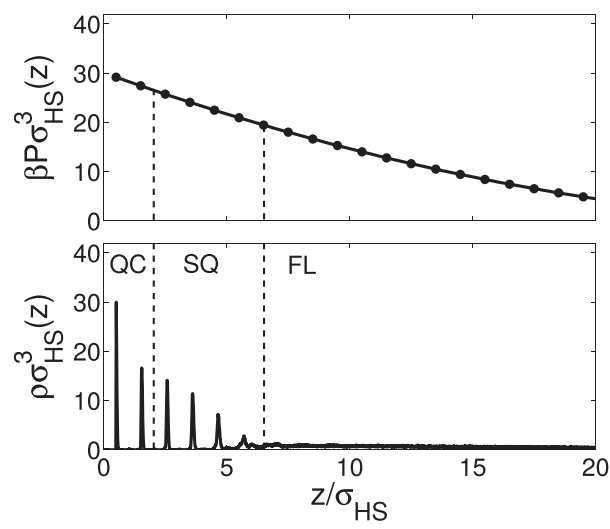

FIG. 8. Pressure (top) and density (bottom) profiles calculated for the QC sedimentation column for Peclet numbers $g^{*}=5.0$ (left) and 2.0 (right) at $t / \tau_{M D}=800$. The stability regions of the dodecagonal quasicrystal (QC), square (SQ), and fluid (FL) phases in terms of the reduced pressure $P_{2 D}^{*}=\beta P \sigma_{H D}^{2}$ as taken from Fig. 1 are marked. 
the layers formed in the sediment exhibit dodecagonal symmetry, which leads to the following question: Why are layers of square symmetry not formed? This could be because of either one of the following reasons: (1) The formation of an interface between the quasicrystal and the periodic crystal might not be energetically favourable or (2) during sedimentation, it might be easier for the particles to settle on top of the particles below because of energetic reasons as explained previously. In addition, a higher settling rate could also contribute to it, i.e., the particles belonging to the top layers do not have sufficient time to rearrange into a square lattice. Also, for the particles to arrange into a square lattice on top of a quasicrystal would mean that the particles of the square layer would fall in the voids of the underlying quasicrystal layer. This again causes an increase in the potential energy. Thus, it is possible that these energetic constraints outweigh those for the formation of a square lattice thereby leading to the formation of only layers with quasicrystal symmetry in the sedimentation column. Finally, we note that although the phase behaviour in 3 -D seems to match well with that in 2-D, the square phase may be absent in 3-D. This will be studied in Sec. III B. It is also good to mention that in the free-energy calculations performed to map out the phase diagram, a defect-free quasicrystal structure was used. It might be possible that the kinetic formation of the quasicrystal with defects precedes that of the defect-free structure. This could also contribute to the formation of the quasicrystal at lower pressures.

Additionally, given that this layered structure of the quasicrystal is not a bulk equilibrium structure, it is interesting to know how many layers of the quasicrystal can be obtained using this sedimentation method. We could estimate this using the hydrostatic equilibrium condition,

$$
d P(z)=-m g \rho(z) d z .
$$

The pressure difference at heights $z_{1}$ and $z_{2}$ is easily obtained by integrating both sides,

$$
\begin{aligned}
\int_{z_{1}}^{z_{2}} d P^{*}(z) & =P^{*}\left(z_{2}\right)-P^{*}\left(z_{1}\right) \\
& =\Delta P^{*}=-\int_{z_{1}}^{z_{2}} g^{*} \rho^{*}(z) d\left(z / \sigma_{H S}\right) .
\end{aligned}
$$

From Fig. 1, we find that the quasicrystal is stable in a pressure range of $\Delta P^{*} \simeq 5$. If we assume the density of the quasicrystal to be constant and independent of height, i.e., $\rho^{*} \simeq 1$, we find that $\Delta P^{*} \simeq 5$ corresponds to a height difference $1 \leq \Delta z \leq 3$ for $g^{*} \in[2,5]$, i.e., 1-3 layers. However, as described above, the quasicrystal was also found to be stable in the stability regime of the square phase. Hence the pressure range increases to $\Delta P^{*} \simeq 20$, which corresponds to 4-10 periodic layers of the quasicrystal. We thus expect to obtain at most 10 periodic layers of the quasicrystal with this method.

\section{B. Formation of layers with square symmetry}

At the end of Sec. III A, we found that it is not feasible for a layer with square symmetry to form on top of a layer with dodecagonal symmetry. Does that mean that the formation of layers with square symmetry is never possible in this system? We address the formation of layers with square symmetry in this section. For this, we first perform sedimentation simulations using the parameters promoting the formation of a square phase as given in Table I and then employ the same analysis methods as those used for studying the quasicrystal formation in the sediment.

First, we show a typical configuration of the sedimentation column obtained at $t / \tau_{M D}=1500$ in Fig. 9(a). The particles are coloured according to the convention in Fig. 2 with particles possessing square symmetry in purple. As can be noticed in the figure, two layers at the bottom of the column have a large concentration of purple coloured particles, denoting the formation of layers with square symmetry. This is further confirmed by the evolution of the BOO $\chi_{4}^{l}$ of the bottom two layers to values close to one, as given in Fig. 9(b). Further, we plot the pressure and density profiles along the sedimentation column, respectively, in Figs. 9(c) and 9(d). From these, we see that the formation of two layers with square symmetry agrees well with the respective phase diagram.

We then follow the dynamics of the formation of these layers by analyzing the snapshots of the bottom two layers as a function of time. The top views of these layers are given in Fig. 10. Similar to the behaviour during the nascent time scales in the QC sediment seen previously in Figs. 4 and 5, the crystal formation in the bottom layer begins with the formation of small crystalline domains of particles with square
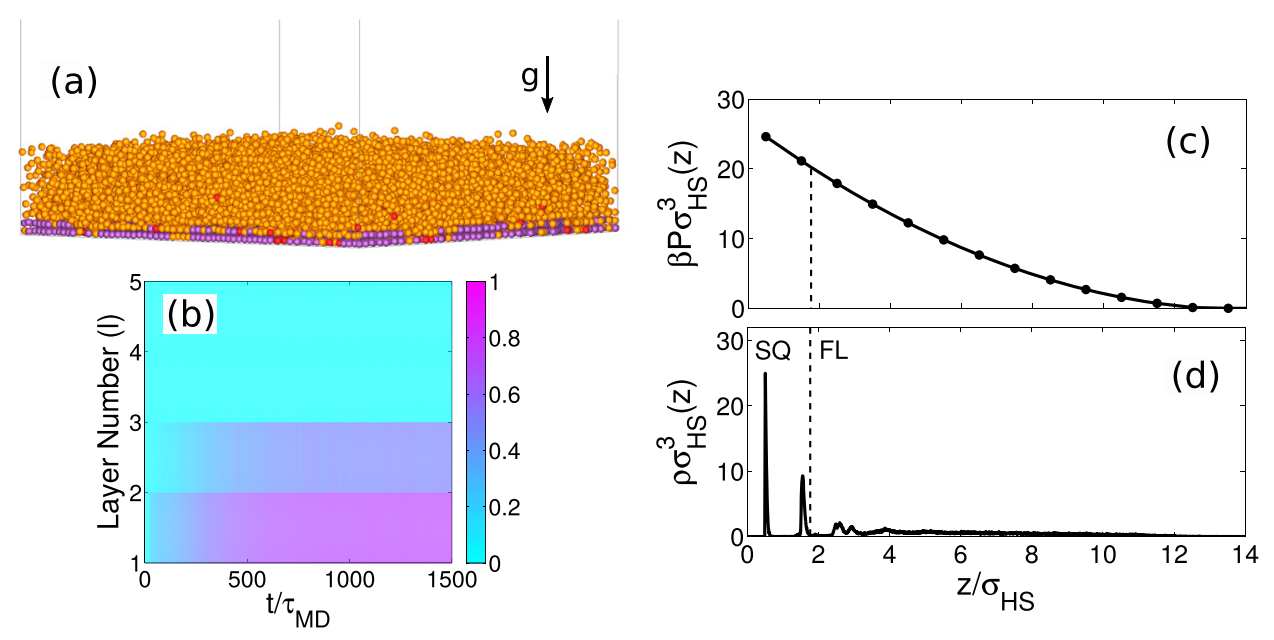

FIG. 9. (a) Side view of a configuration of the SQ sedimentation column obtained at $t / \tau_{M D}=1500$. The particles are coloured according to their individual BOO: quasicrystal (red), square (purple), hexagon (green), and fluid (orange). (b) The $\mathrm{BOO} \chi_{4}^{l}$ of each layer as a function of time showing the formation of layers with square symmetry. (c) Pressure and (d) density profiles calculated for the sedimentation column at $t / \tau_{M D}=1500$. The stability regions of square (SQ) and fluid (FL) phases in terms of reduced pressure $P_{2 D}^{*}=\beta P \sigma_{H D}^{2}$ as taken from Fig. 1 are marked. 

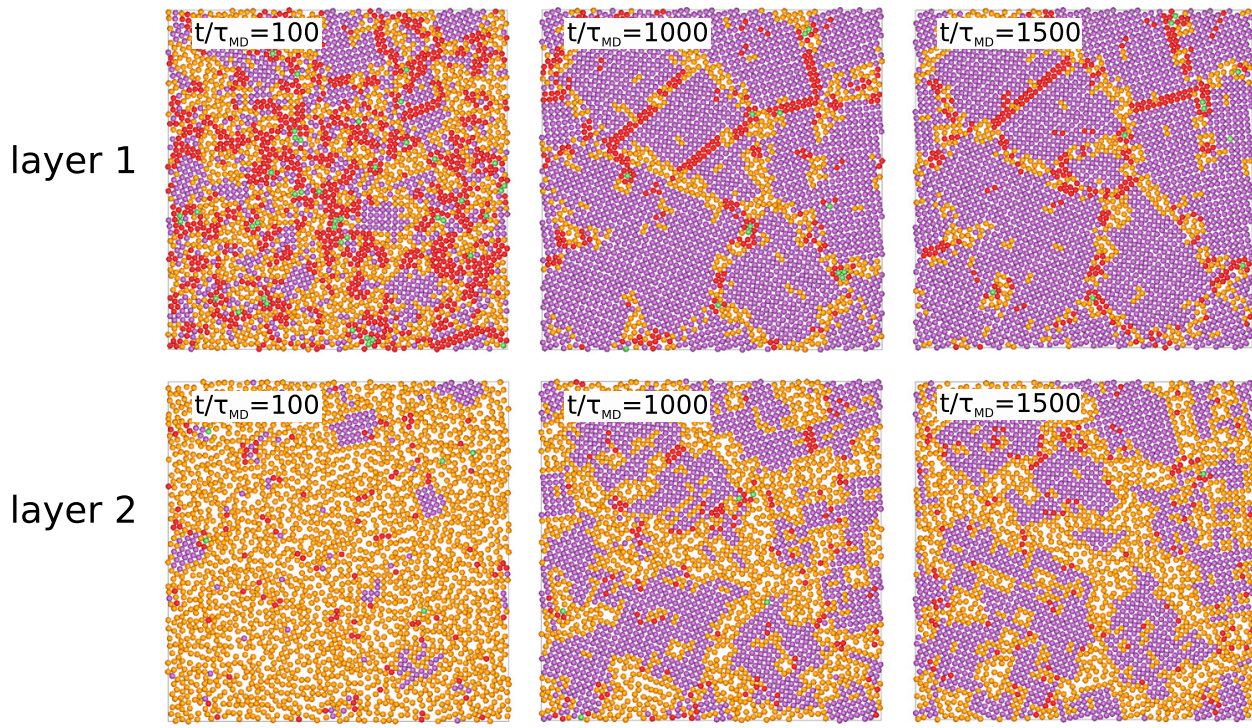

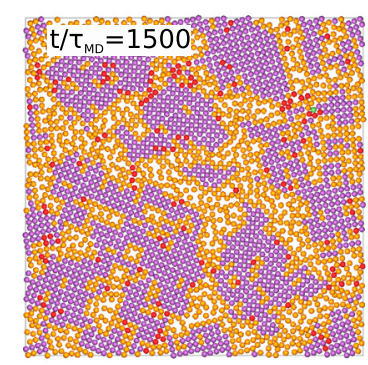

FIG. 10. Typical configurations showing the top view of the first (top) and second (bottom) layers of the SQ sediment for varying times $t / \tau_{M D}$ as labeled. The particles are coloured according to their individual BOO, namely, quasicrystal (red), square (purple), hexagonal (green), and fluid (orange). layer 2

symmetry $\left(t / \tau_{M D}=100\right)$. These crystalline domains coalesce with time and form larger grains separated by grain boundaries consisting of fluid-like particles or particles with dodecagonal symmetry $\left(t / \tau_{M D}=1000\right)$. Finally, these grain boundaries anneal out with time $\left(t / \tau_{M D}=1500\right)$. Obviously, the settling rate plays an important role in the annealing process. In the present scenario, we find that the second layer starts to crystallise before the grain boundaries in the bottom layer are annealed out. Thus, these grain boundaries remain till the end of our simulation. Additionally, we observe that this structure also consists of periodic layers, with the particles of the second layer lying on top of the ones in the first layer.

\section{Formation of (suspended) layers with hexagonal symmetry}

One of the peculiar features exhibited by the HCSS system is the formation of a low-density hexagonal phase, where the particles are separated by a distance equal to the square shoulder diameter $\delta$. The formation of this phase induces a re-entrant behaviour of the fluid phase. Our objective in this section is two-fold. We attempt to (1) demonstrate the formation of layers of the low-density hexagonal phase and analyze if it conforms to either a face-centered cubic (FCC), a hexagonal close-packed (HCP), or a AAA stacking and (2) determine if the fluid displays a re-entrant behaviour along the height of the sedimentation column, i.e., with decreasing pressure. This would result in a configuration where hexagonal layers are suspended in between two fluid phases, i.e., a floating crystal phase.

Accordingly, we first simulate the sedimentation of particles with parameters that promote the formation of a lowdensity hexagonal phase as given in Table I. A typical configuration of the sedimentation column at $t / \tau_{M D}=500$ is given in Fig. 11(a). The particles here are coloured according to the convention given in Fig. 2. Correspondingly, the layers at the bottom composed of particles coloured in green exhibit hexagonal symmetry. The presence of hexagonal symmetry of these layers is confirmed by the high values of the $\chi_{6}^{l}$ values calculated as a function of time for these layers and plotted in
Fig. 11(b). Combining this time evolution of BOO and the pressure [Fig. 11(c)] and density [Fig. 11(d)] profiles along the sedimentation column, we find the formation of about twenty layers of hexagonal symmetry. This also agrees well with the pressure boundaries obtained from the phase diagram, marked with dashed lines in Figs. 11(c) and 11(d). Further, we try to analyze the composition of these hexagonal layers to identify the nature of the stacking. It is common to find the formation of randomly stacked hexagonal layers in experimental and simulation sedimentation studies on hard spheres conducted at low Peclet numbers. ${ }^{21,22}$ This is because the free-energy difference between the face-centered cubic (FCC) and hexagonal close-packed (HCP) phases is very small in the case of hard spheres. We calculate the nature of stacking by using the method described by Marechal et al. ${ }^{21}$ We represent this by colour coding layers stacked as FCC in dark red and HCP in blue in Fig. 11(e). We observe a behaviour analogous to that of a system of hard spheres, i.e., the formation of randomly stacked hexagonal layers. However, we did not find any slanted stacking faults as observed in previous EDBD simulations on sedimenting hard spheres. ${ }^{21,22}$ We also note that in contrast to the quasicrystal and the square crystals, we do not find that the hexagonal layers are periodically stacked.

We then proceed to explore the formation of suspended crystalline layers of hexagonal symmetry bordered by a fluid phase in the sediment. For this, we use a set of parameters conforming to the formation of a fluid phase at the bottom of the sedimentation column as given in Table I. Following the convention, we first show a typical configuration of the sedimentation column at $t / \tau_{M D}=600$ in Fig. 12(a) and colour the particles according to their BOO as explained in Fig. 2. We indeed find the formation of crystalline layers with hexagonal symmetry in between two fluid phases. In other words, we observe a re-entrant behaviour of the fluid phase bordering a floating crystal phase with layers of hexagonal symmetry. The hexagonal symmetry of the layers in the crystal phase is further proven by the high $\chi_{6}^{l}$ BOO values shown in the time evolution heat map in Fig. 12(b). However, we observe that the corresponding pressure [Fig. 12(d)] and density [Fig. 12(c)] 

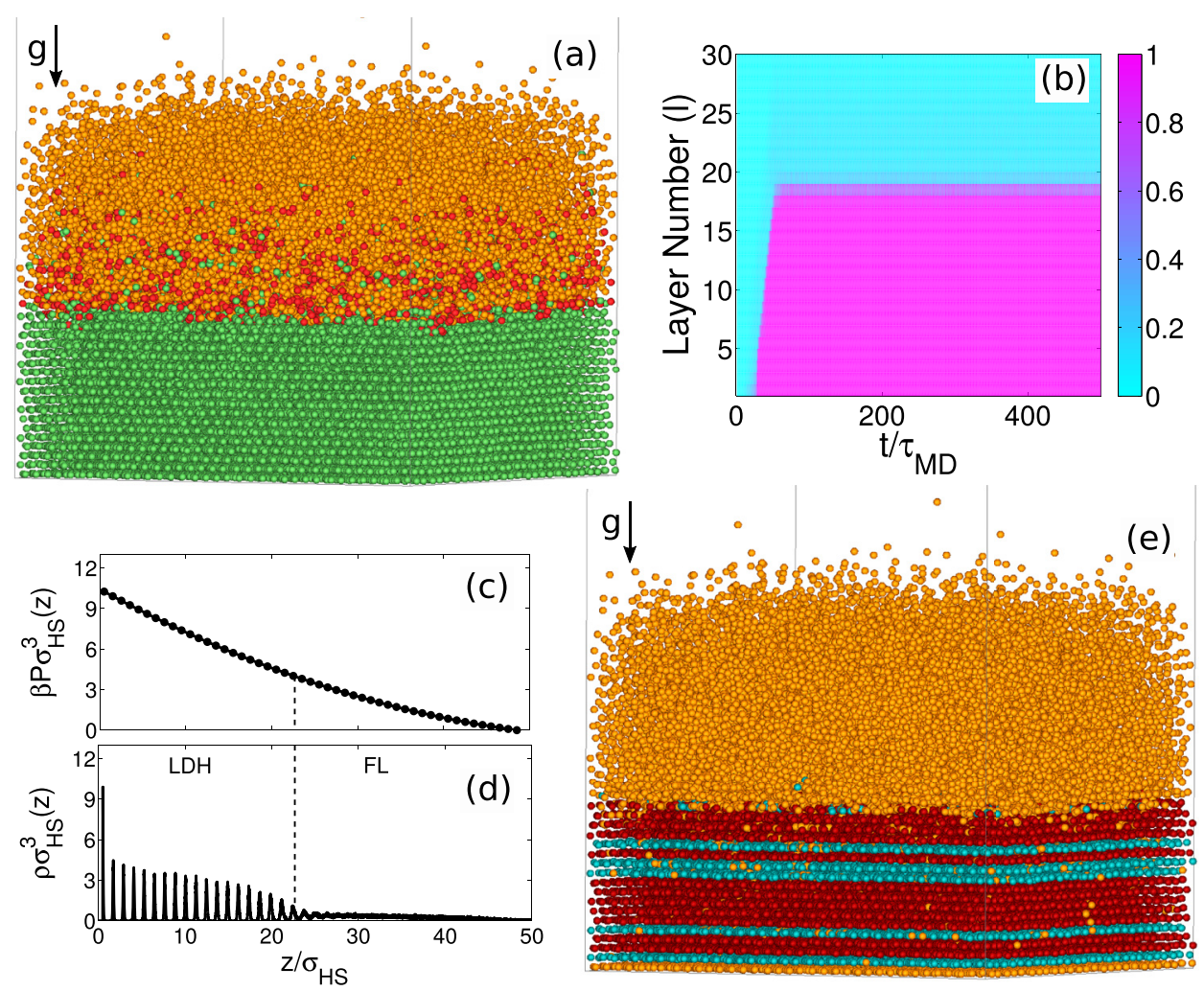

FIG. 11. (a) Side view of a configuration of the low-density hexagonal (LDH) sedimentation column obtained at $t / \tau_{M D}=500$. The particles are coloured according to their individual BOO: quasicrystal (red), square (purple), hexagon (green), and fluid (orange). (b) The $\mathrm{BOO} \chi_{6}^{l}$ of each layer as a function of time showing the formation of layers with hexagonal symmetry. (c) Pressure and (d) density profiles calculated along the height of the sedimentation column. The stability regions of low-density hexagonal (LDH) and fluid (FL) phases in terms of reduced pressure $P_{2 D}^{*}=\beta P \sigma_{H D}^{2}$ as taken from Fig. 1 are marked. (e) Identification of layers as FCC or HCP stacking in the sediment. The particles are coloured as FCC (red) and HCP (blue). profiles of the sediment have not yet completely evolved. Also, similar to the previous case of hexagonal layers, we find that these suspended layers are also randomly stacked.

The interesting feature of this sedimentation process is the formation of alternate regions of hexagonal symmetry and fluid at the bottom of the sediment with time. This can be observed in the time evolution of the hexagonal BOO $\chi_{6}^{l}$ in Fig. 12(b). At the start of the sedimentation process, the sedimentation column is filled with an isotropic fluid, as seen by the low value of $\chi_{6}^{l}$ in blue. With time, the density at the bottom increases.

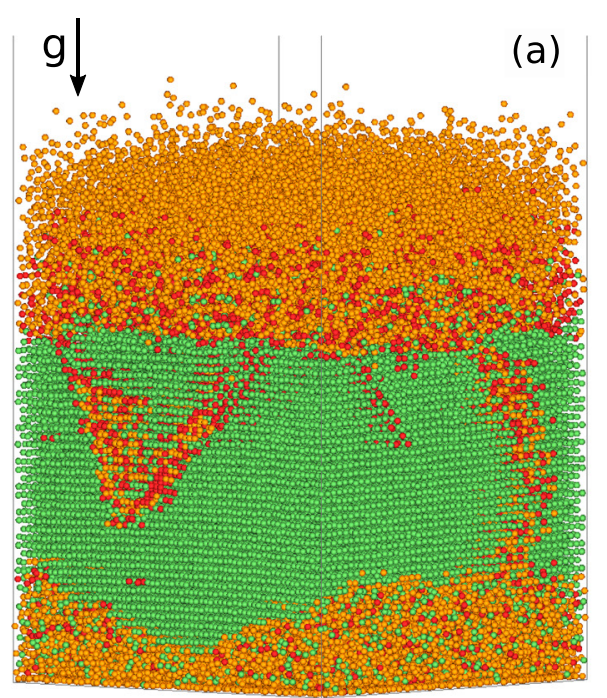

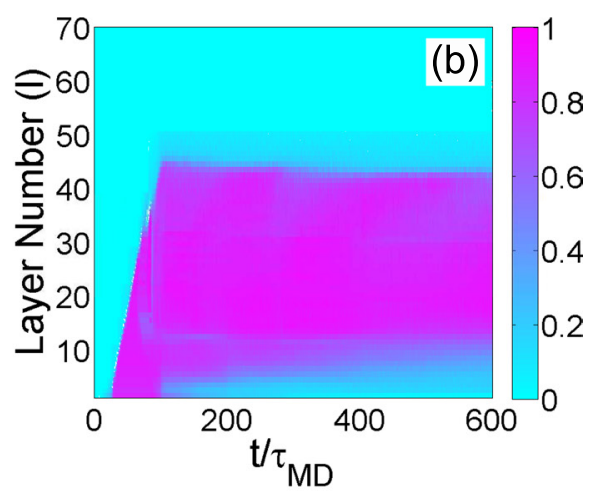

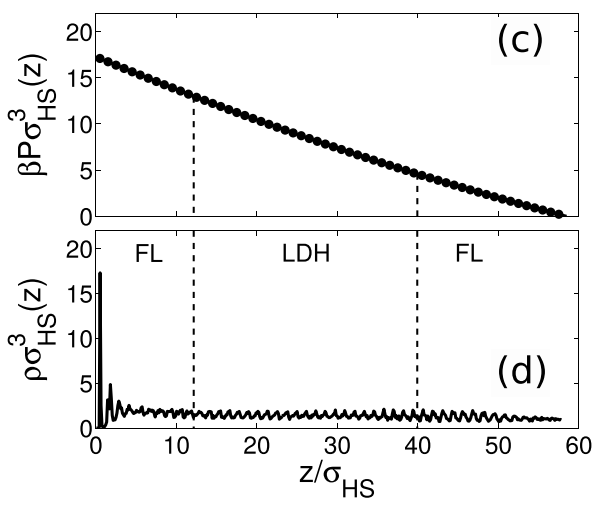

FIG. 12. (a) Side view of a configuration of the FL sedimentation column obtained at $t / \tau_{M D}=500$. The particles are coloured according to their individual BOO: quasicrystal (red), square (purple), hexagon (green), and fluid (orange). (b) The BOO $\chi_{6}^{l}$ of each layer as a function of time showing the formation of layers with hexagonal symmetry. (c) Pressure and (d) density profiles calculated along the height of the sedimentation column. The stability regions of low-density hexagonal (LDH) and fluid (FL) phases in terms of reduced pressure $P_{2 D}^{*}=\beta P \sigma_{H D}^{2}$, as taken from Fig. 1, are marked. 

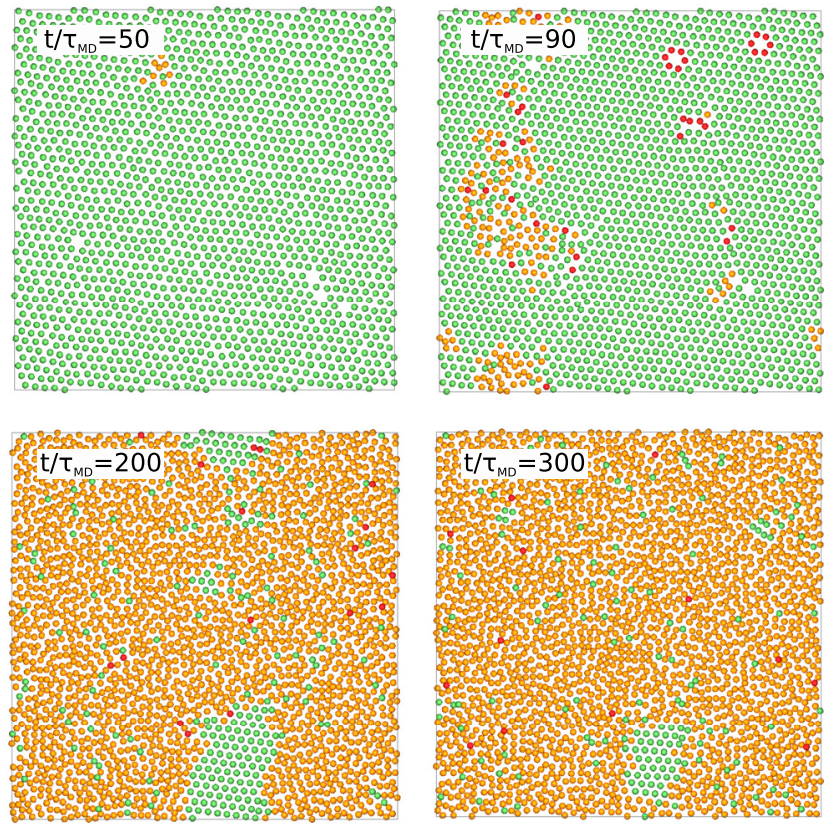
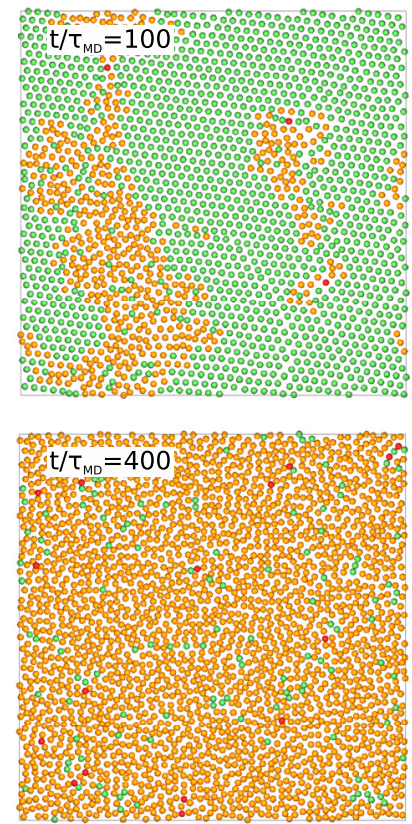

FIG. 13. Top view of the first layer of the sediment showing the melting of the hexagonal phase to a fluid for varying $t / \tau_{M D}$ as labeled. The particles are coloured according to their individual BOO, namely, quasicrystal (red), square (purple), hexagon (green), and fluid (orange).
Once it reaches the density where the hexagonal phase is found to be stable, structures with hexagonal symmetry start to form at the bottom layers. This is seen as an increase in the values of $\chi_{6}^{l}$ represented by pink colour. With time, we observe that more hexagonal layers start to form. In contrast, we also see that the pink colour at the bottom gives way to the blue colour again denoting the melting of the hexagonal layers into a fluid with an increase in the pressure. We also observe more layers melting as time proceeds. This melting process of the first layer of the sediment can also be seen from the particle configurations taken at different times, which is shown in Fig. 13. We observe the formation of larger fractions of fluid with time in these snapshots.

\section{CONCLUSIONS AND OUTLOOK}

To summarise, we studied the sedimentation behaviour of a system of particles interacting with a hard-core and a repulsive square shoulder potential with a fixed shoulder width equal to 1.4 times the hard-core diameter. We find that the system forms a two-dimensional layered structure because of energetic arguments. This enables us to validate the formation of the thermodynamically stable phases as predicted by the two-dimensional phase diagram, which is highly surprising. Accordingly, we confirm that layers with symmetries of a dodecagonal quasicrystal, square, and low-density hexagonal are formed in the pressure range corresponding to the respective stability regions in the 2-D phase diagram. Further ascertaining the validity of the calculated phase diagram, we find that the fluid phase, in the case of a low-density hexagonal phase, exhibits a re-entrant phase behaviour along the height of the sedimentation column. In addition, we evaluated the effect of settling rates on the formation of the quasicrystal and found that faster settling rates lead to the formation of a quasicrystal with a tiling composition closer to that of the maximum entropy tiling.
For future work, it is interesting to study the formation of the dodecagonal quasicrystal in detail and to determine the optimal pressure and settling rates for its formation. It is also interesting to see how the quasicrystal formation is affected in the case of colloidal epitaxy.

\section{ACKNOWLEDGMENTS}

This work is part of the Industrial Partnership Programme "Computational Sciences for Energy Research" (Grant No. 12CSER004) of the Foundation for Fundamental Research on Matter (FOM), which is part of the Netherlands Organisation for Scientific Research (NWO). This research programme is co-financed by Shell Global Solutions International B.V. We thank John R. Edison for providing the EDBD code used in this work, Tonnishtha Dasgupta for calculating the stacking of the hexagonal layers (Fig. 11) and for critical reading of this manuscript, and Wessel S. Vlug for performing the tiling calculations.

${ }^{1}$ J. G. Kirkwood, E. K. Maun, and B. Alder, J. Chem. Phys. 18, 1040 (1950).

${ }^{2}$ R. Piazza, T. Bellini, and V. Degiorgio, Phys. Rev. Lett. 71, 4267 (1993).

${ }^{3}$ A.-P. Hynninen, R. van Roij, and M. Dijkstra, Europhys. Lett. 65, 719 (2004).

${ }^{4}$ T. Biben and J.-P. Hansen, Mol. Phys. 80, 853 (1993).

${ }^{5}$ S. Savenko and M. Dijkstra, Phys. Rev. E 70, 051401 (2004).

${ }^{6}$ X. Zhang, Z.-Q. Zhang, and C. Chan, Phys. Rev. B 63, 081105 (2001).

${ }^{7}$ M. C. Rechtsman, H. C. Jeong, P. M. Chaikin, S. Torquato, and P. J. Steinhardt, Phys. Rev. Lett. 101, 073902 (2008).

${ }^{8}$ K. Edagawa, Sci. Technol. Adv. Mater. 15, 034805 (2014).

${ }^{9}$ A. Micco, V. Galdi, F. Capolino, A. Della Villa, V. Pierro, S. Enoch, and G. Tayeb, Phys. Rev. B 79, 075110 (2009).

${ }^{10}$ S. M. Thon, W. T. M. Irvine, D. Kleckner, and D. Bouwmeester, Phys. Rev. Lett. 104, 243901 (2010).

${ }^{11}$ A. Ricciardi, M. Pisco, A. Cutolo, A. Cusano, L. O'Faolain, T. F. Krauss, G. Castaldi, V. Galdi, L. O'Faolain, T. F. Krauss, G. Castaldi, and V. Galdi, Phys. Rev. B 84, 085135 (2011).

${ }^{12}$ M. Shimoda, T. Sato, A. Tsai, and J. Guo, Phys. Rev. B 62, 11288 (2000).

${ }^{13}$ K. Franke, H. Sharma, W. Theis, P. Gille, P. Ebert, and K. Rieder, Phys. Rev. Lett. 89, 156104 (2002). 
${ }^{14}$ V. Fournée, T. Cai, A. Ross, T. A. Lograsso, J. W. Evans, and P. A. Thiel, Phys. Rev. B 67, 033406 (2003).

${ }^{15}$ S. Curtarolo, W. Setyawan, N. Ferralis, R. D. Diehl, and M. W. Cole, Phys. Rev. Lett. 95, 136104 (2005).

${ }^{16}$ B. Bilki, M. Erbudak, M. Mungan, and Y. Weisskopf, Phys. Rev. B 75, 045437 (2007).

${ }^{17}$ M. Mungan, Y. Weisskopf, and M. Erbudak, Phys. Rev. B 76, 195443 (2007).

${ }^{18}$ M. Schmiedeberg and H. Stark, Phys. Rev. Lett. 101, 218302 (2008).

${ }^{19}$ H. Pattabhiraman, A. P. Gantapara, and M. Dijkstra, J. Chem. Phys. 143, 164905 (2015).

${ }^{20}$ H. Pattabhiraman and M. Dijkstra, J. Phys.: Condens. Matter 29, 094003 (2017).

${ }^{21}$ M. Marechal, M. Hermes, and M. Dijkstra, J. Chem. Phys. 135, 034510 (2011).
${ }^{22}$ T. Dasgupta, J. R. Edison, and M. Dijkstra, J. Chem. Phys. 146, 074903 (2017).

${ }^{23}$ J. P. Hoogenboom, P. Vergeer, and A. van Blaaderen, J. Chem. Phys. 119, 3371 (2003).

${ }^{24}$ H. Pattabhiraman and M. Dijkstra, Soft Matter 13, 4418 (2017).

${ }^{25}$ M. Engel, J. A. Anderson, S. C. Glotzer, M. Isobe, E. P. Bernard, and W. Krauth, Phys. Rev. E 87, 042134 (2013).

${ }^{26}$ L. Fernandez, V. Martín-Mayor, B. Seoane, and P. Verrocchio, Phys. Rev. Lett. 108, 165701 (2012).

${ }^{27} \mathrm{H}$. Weber, D. Marx, and K. Binder, Phys. Rev. B 51, 14636 (1995).

${ }^{28}$ T. Dotera, T. Oshiro, and P. Ziherl, Nature 506, 208 (2014).

${ }^{29}$ M. Widom, Phys. Rev. Lett. 70, 2094 (1993).

${ }^{30}$ M. Baake, R. Klitzing, and M. Schlottmann, Phys. A 191, 554 (1992). 